\title{
PROPUESTA DE ACCIONES PARA EL MEJORAMIENTO DEL DESEMPEÑO AMBIENTAL EN ENTIDADES DE RESTAURACIÓN.
}

\section{PROPOSED ACTIONS FOR IMPROVEMENT OF ENVIRONMENTAL PERFORMANCE IN RESTAURANT ENTITIES.}

Martha Omara Robert Beatón, MSc. Máster en Gestión Turística (Cuba). Vicedecana de Trabajo Educativo de la Facultad de Turismo en la Universidad de La Habana, Cuba. omara_robert@ftur.uh.cu

Maité Echarri Chávez, PhD. Doctora en Ciencias Geográficas (Cuba). Coordinadora de la Maestría en Gestión Turística en la Universidad de La Habana, Cuba. maite_echarri@ftur.uh.cu

\section{ARTÍCULO DE INVESTIGACIÓN}

Recibido: 22 de octubre de 2016.

Aceptado: 21 de diciembre de 2016.

\section{RESUMEN}

La investigación que se presenta tiene como objetivo proponer acciones para el mejoramiento del desempeño ambiental en entidades de restauración del destino La Habana. Los métodos empleados fueron el análisis y síntesis de la bibliografía sobre el tema objeto de estudio y el análisis del desempeño ambiental para cada una de las instalaciones seleccionadas, a partir de una guía de observación ambiental propuesta en investigaciones precedentes. Los resultados de este análisis permitieron, identificar las principales debilidades y limitaciones que inciden en el desempeño ambiental de las entidades en cuestión, y a partir de ello proponer acciones dirigidas a sentar las bases para la obtención de los distintos reconocimientos ambientales y para la futura implementación de los sistemas de gestión ambiental en restaurantes del destino La Habana. 
Palabras clave: gestión medioambiental, desempeño ambiental, entidades de restauración, turismo.

\section{ABSTRACT}

The investigation that presents itself aims at proposing stock for the improvement of the environmental performance at entities of restoration of the destination Havana. Used methods were the analysis and I object synthesis of the topical bibliography of study and the environmental analysis of performance for each of facilities selected, as from a guide of environmental observation proposed in preceding investigations. The results of this analysis permitted, identifying the principal weaknesses and limitations that have an effect on entities' in point environmental performance, and as from it proposing guided stock to seat the bases for the obtaining of distinct environmental recognitions for the future implementation of the systems of environmental steps and at restaurants of the destination Havana.

Keywords: environmental management, environmental acting, restoration entities, tourism.

\section{INTRODUCCIÓN}

La gestión ambiental para las entidades turísticas cubana resulta hasta el presente un proceso poco valorado en cuanto a los impactos positivos que genera para la gestión propia y de cara a clientes y prestatarios. Disponer de certificaciones, sellos y avales ambientales no ha encontrado en el panorama turístico cubano receptores motivados que los visualicen como valores agregados, elementos distintivos en un entorno cada vez más competitivo y donde la búsqueda de propuestas forma parte de las iniciativas que pueden ser impulsadas desde las propias instalaciones.

En Cuba, la mayoría de los estudios han estado encaminados a identificar las causas y efectos de los problemas ambientales en correspondencia con lo legislado por el Ministerio de Ciencia Tecnología y Medio Ambiente, y al mismo tiempo a trazar estrategias que permitan frenar la acelerada e irracional explotación de los recursos naturales y la evaluación sobre su gestión. Las normativas incluidas en la NC 14000 y su familia, han permitido hasta cierto punto encauzar las acciones a realizar pero aún queda mucho camino por delante, siendo insuficientes los estudios que aborden propuesta de acciones para el mejoramiento del desempeño ambiental desde los diferentes espacios del sector turístico, que logren certeramente la integración del medio ambiente en los 
procesos de desarrollo, a partir de parámetros de consumo ambientalistas, y del logro de un desarrollo cada vez más sostenible y equilibrado socialmente.

Es por ello que la Facultad de Turismo de la Universidad de La Habana dirige uno de sus proyectos de investigación hacia la evaluación de la gestión ambiental en entidades del sector. Antecedentes pueden encontrarse en trabajos de investigación, fundamentalmente ejercicios de culminación de estudios, que han abordado dicha temática en los restaurantes La vicaria (Díaz, 2013), El Delfín (Águila, 2015), La Casa Española (Marín, 2015) y El Cochinito (Cabanes, 2014) precisando la realización de un compendio que permita la comparación de las actuaciones en materia ambiental del GEE Palmares, Cimex y Restaurantes de La Habana, casas matrices de los restaurantes mencionados. Ante la multiplicidad de enfoques, en cuanto a la gestión ambiental en los diferentes restaurantes tomados como objeto de estudio, así como las diferencias reflejadas en los resultados alcanzados entre las investigaciones realizadas siguiendo la misma guía de evaluación se hace necesario proponer acciones que permitan la estandarización, hacia el establecimiento de normas y buenas prácticas de manejo para el mejoramiento de la gestión ambiental en función de un desarrollo organizacional sostenible, por lo que se plantea la siguiente problema de investigación: No se han sistematizado los resultados derivados de investigaciones precedentes para la propuesta de acciones de mejoramiento del desempeño ambiental de entidades de restauración del destino La Habana, a partir del cual se define como objetivo de la presente investigación: Proponer acciones para el mejoramiento del desempeño ambiental en entidades de restauración del destino La Habana.

\section{REVISIÓN TEÓRICA}

\section{El paradigma ambiental: de la teoría a la práctica.}

La gestión ambiental se define como el conjunto de actividades, mecanismos, acciones e instrumentos, dirigidos a garantizar la administración y uso racional de los recursos naturales mediante la conservación, mejoramiento, rehabilitación y monitoreo del medio ambiente y el control de la actividad del hombre en esta esfera. La gestión ambiental aplica la política ambiental establecida mediante un enfoque multidisciplinario, teniendo en cuenta el acervo cultural, la experiencia nacional acumulada y la participación ciudadana (Ley 81, 1997).

Organizaciones de todo tipo están cada vez más interesadas en alcanzar y demostrar un sólido desempeño ambiental mediante el control de los impactos de sus actividades, 
productos y servicios sobre el medio ambiente, acorde con su política y objetivos ambientales. Lo hacen en el contexto de una legislación cada vez más exigente, del desarrollo de políticas económicas y otras medidas para fomentar la protección ambiental, y de un aumento de la preocupación expresada por las partes interesadas por los temas ambientales, incluido el desarrollo sostenible (Hernández, 2007).

A partir del año 1996 la ISO (Organización Internacional de Normalización) desarrolló un grupo de normas internacionales relacionadas con la gestión ambiental y que se conoce ampliamente como las normas de la familia ISO 14000. Esta familia de normas, Cuba las adopta como normas cubanas con las siglas NC ISO 14000.

La ISO 14001 es el documento de referencia empleado internacionalmente, para otorgar el distintivo por los Órganos Certificadores de Sistemas de Gestión Ambiental (SGA). El objetivo general de este modelo es apoyar, la protección ambiental y la prevención de la contaminación en equilibrio con las necesidades socio-económicas. En particular, la NC ISO 14001 en su versión más actualizada del año 2004 específica los requisitos para un sistema de gestión ambiental que le permita a una organización desarrollar e implementar una política y unos objetivos que tengan en cuenta los requisitos legales y la información sobre los aspectos ambientales significativos. Es aplicable a todos los tipos y tamaños de organizaciones y se ajusta a diversas condiciones geográficas, culturales y sociales.

Sin embargo, la NC ISO 14001 no garantiza en sí misma unos resultados ambientales óptimos. Para lograr objetivos ambientales, el sistema de gestión ambiental puede estimular a las organizaciones a considerar la implementación de las mejores técnicas disponibles cuando sea apropiado y económicamente viable, y a tener en cuenta completamente la relación entre el costo y la eficacia de estas técnicas (Almeida, 2004).

A fin de prevenir la contaminación, surgen en la actualidad, herramientas en estos SGA que suelen ser implementados por las organizaciones como es el empleo de producciones más limpias. Estas pueden definirse como: la aplicación de una estrategia ambiental preventiva e integrada aplicada a los procesos productivos, productos y servicios según PNUMA (2000) e incluyen la conservación de materias primas y energía, la eliminación de materias primas tóxicas, la reducción en la cantidad y toxicidad de las emisiones y desechos, un uso más eficiente de los recursos naturales, minimiza los desechos y la contaminación así como el riesgo a la salud humana y a la seguridad. En resumen, las producciones más limpias ofrecen muchas ventajas en el mejoramiento continuo referente al medio ambiente, (CIGEA, 2002).

Los elementos a considerar en un SGA deben comprometer a la entidad turística a su 
mejoramiento continuo, debe constituir un significativo aporte a la Seguridad y Calidad de los productos y servicios que se ofertan a los clientes, factor determinante para el desarrollo y fortalecimiento gradual de los resultados. Los mismos deben estar en consonancia con los principios del Desarrollo Sostenible, de manera tal que los procesos, productos y servicios se conciban y realicen en armonía con el medio ambiente, contribuyendo a su conservación para las presentes y futuras generaciones. Es muy importante que logren el desarrollo de la Ciencia y la Innovación Tecnológica integrada objetivamente con las actividades de Seguridad y Calidad para obtener eficientes resultados y así como la reducción constante de los impactos ambientales que se puedan generar producto del desempeño. Además deben proyectar el logro de procesos productivos más limpios con el fin de disminuir los consumos y hacer un uso eficiente de los recursos disponibles, para contribuir a la disminución de la carga contaminante, sumada a la caracterización y control constante de todos los desechos a través de mediciones, evaluaciones y una adecuada deposición de los mismos. Deben enfocar el trabajo en la prevención y disminución al mínimo de los riesgos y ocurrencia de accidentes e incidentes que puedan ocasionar posibles daños a cualquiera de las partes interesadas. Deben implicar la Gestión del Conocimiento para formar e informar conscientemente a los trabajadores y clientes, de manera que se incremente sistemáticamente la educación y cultura medioambiental (Tejeda, 2010).

El alcance de la gestión ambiental de las entidades turísticas depende de sus capacidades y recursos, y no se debe limitar a aquellos elementos de sus actividades, productos y servicios que son previamente considerados de mayor prioridad por la dirección. Con el tiempo, este alcance puede ampliarse e ir incorporando paulatinamente elementos de las actividades, productos y servicios de la organización que no se habían considerado anteriormente.

Las exigencias ambientales de los consumidores turísticos adquieren cada día una especial relevancia como motores de un cambio en las diversas formas de la actividad turística. Este distintivo de la calidad ambiental en las organizaciones comienza a ser una exigencia para el negocio turístico de avanzada. Hoy una certificación ambiental representa un factor diferenciador de oferta; por tanto, las empresas que tributan con sus productos y servicios tienen que estar acordes con estas exigencias. De todo lo anterior se infiere la necesidad de un adecuado programa de educación ambiental, dirigido hacia todos los empleados de la empresa turística. Es por ello que el tema medio ambiente en el contexto turístico, ha pasado a ocupar un lugar de importancia. Dentro de este 
movimiento proambientalista, las empresas turísticas desarrollan diversas vías para contribuir a este objetivo, constituyendo la gestión ambiental, una de las actividades prioritarias en esta dimensión de trabajo.

En medio de este contexto, emergen los establecimientos de restauración como ejemplos concretos del despliegue de acciones en la búsqueda de lograr un equilibrio entre el desarrollo turístico y el cuidado del medio ambiente, siendo los Sistemas de Gestión Ambiental (SGA) la herramienta principal empleada por estas entidades.

La práctica y los estudios en este sentido han demostrado que, si bien se referencia al mismo en las estrategias gerenciales de la mayoría de las instalaciones de restauración, las acciones que se realizan en este sentido y los beneficios que arrastra, no se perciben nítidamente por los clientes e incluso por el personal y directivos de la instalación.

El tratamiento y solución a esta situación, pasa, obligatoriamente, por entender el fenómeno perceptivo, desde la perspectiva de la gestión ambiental, de modo que facilite trasmitir la importancia del fenómeno medioambiental en la restauración, su necesidad y utilidad, tanto a efectos de la actividad como del propio entorno, buscando lograr que todas las partes implicadas se hagan partícipe y valoren la cuestión en toda su magnitud.

\section{MATERIALES Y MÉTODOS}

Evaluación del desempeño ambiental: normas y procedimientos.

De acuerdo a lo planteado por González (2006), las evaluaciones ambientales se deben valorar dentro de la concepción holística del medio ambiente, tomando en consideración las políticas, programas, planes y proyectos de desarrollo. La evaluación ambiental nace como una herramienta de protección ambiental que, apoyada por la institucionalidad acorde a las necesidades de las distintas organizaciones, fortalece la toma de decisiones a nivel de políticas, planes, programas y proyectos (Maza, 2009).

Según la Norma Cubana ISO 14031:2005 se define el desempeño ambiental como los resultados de la gestión de una organización sobre sus aspectos ambientales y la Evaluación del Desempeño Ambiental (EDA) como un proceso utilizado para facilitar las decisiones de la dirección con respecto al desempeño ambiental de la organización mediante la selección de indicadores, la recopilación y el análisis de datos, la evaluación de la información comparada con los criterios de desempeño ambiental, los informes y comunicaciones, las revisiones periódicas y las mejoras de este proceso.

La EDA sigue el modelo de gestión "Planificar-Hacer-Verificar-Actuar". A continuación se describen los pasos de este proceso continuo: 
a) Planificar

1) Planificación de la EDA. (Evaluación del Desempeño Ambiental)

2) Selección de indicadores para la EDA (el proceso de selección de indicadores puede incluir tanto la selección de los indicadores existentes como el desarrollo de nuevos indicadores).

b) Hacer Uso de datos e información que incluye:

1) La recopilación de los datos pertinentes para los indicadores seleccionados.

2) El análisis y la conversión de los datos en información que describa el desempeño ambiental de la organización.

3) La evaluación de la información que describe el desempeño ambiental de la organización en comparación con sus criterios de desempeño ambiental.

4) El informe y la comunicación de la información que describa el desempeño ambiental de la organización.

c) Verificar y actuar. La revisión y mejora de la EDA. La EDA es un proceso interno de gestión que utiliza indicadores para proporcionar información, comparando el desempeño ambiental pasado y presente de una organización con sus criterios de desempeño ambiental. Existen dos tipos de indicadores: del desempeño ambiental y de la condición ambiental. Los indicadores del desempeño ambiental pueden ser indicadores del desempeño de gestión, los cuales proporcionan información sobre el esfuerzo de la dirección para influir en el desempeño ambiental de las operaciones de la organización; y además pueden ser indicadores del desempeño operacional que proporcionan información sobre el desempeño ambiental de las operaciones de la organización. Los indicadores de la condición ambiental proporcionan información sobre la condición ambiental. Esta información puede ayudar a una organización a comprender el impacto real o potencial de sus aspectos ambientales. Una organización con un sistema de gestión ambiental implementado debería evaluar su desempeño ambiental respecto a su política ambiental, objetivos, metas y otros criterios de desempeño ambiental. Cuando una organización no cuenta con un sistema de gestión ambiental, la EDA puede ayudar a la organización en:

- La identificación de sus aspectos ambientales.

- La determinación de qué aspectos serán tratados como significativos.

- El establecimiento de criterios para su desempeño ambiental.

- La evaluación de su desempeño ambiental frente a estos criterios. 
La Evaluación del Desempeño Ambiental (EDA) es un proceso y una herramienta de gestión interna diseñada para proporcionar continuamente a la dirección información fiable y verificable para determinar si el desempeño ambiental de una organización está cumpliendo con los criterios establecidos por la dirección de dicha organización. La información generada por la EDA puede ayudar a la organización a:

- Determinar cualquier acción necesaria para alcanzar sus criterios del desempeño ambiental.

- Identificar aspectos ambientales significativos.

- Identificar oportunidades para mejorar la gestión de sus aspectos ambientales (por ejemplo, la prevención de la contaminación)

- Identificar tendencias en su desempeño ambiental.

\section{METODOLOGÍA}

El proceso metodológico se desarrolló en 3 fases, siendo la primera la elaboración de la guía de observación ambiental que permitiera su generalización para la evaluación del desempeño ambiental en otras entidades de restauración. Para la elaboración de la Guía de Observación Ambiental (GOA) se tuvieron en cuenta las fases de trabajo siguientes:

1. Recopilación y análisis de la documentación referente a la problemática medioambiental, relacionada con:

a) Lista de chequeo del Programa Cocina + Higiénica y segura (de la Paz Prieto, 2011);

b) Guía de observación de la NC 126 (ONN, 2001);

c) Cuestionario de aspectos ambientales para la aplicación práctica de los sistemas de gestión ambiental (Tejeda Montero, M., 2010);

d) Estrategia ambiental. Actualización para el período 2013-2015 (MINTUR, 2012), de los cuales se tomaron aquellos aspectos vinculados con la actividad de restauración desde el punto de vista medioambiental y que eran factibles de aplicar, es decir que resultaran estándar para cualquier categoría de restaurante de modo que facilitara su posterior generalización.

2. Observación in situ del desempeño medioambiental del restaurante: teniendo en cuenta que la experiencia práctica posibilita un mejor criterio de evaluación se observaron las dinámicas asociadas a las actividades siguientes: los flujos de servicio, el comportamiento del personal, las condiciones de la instalación, así como determinados factores que 
incidían en el funcionamiento del restaurante; también se realizaron intercambios con trabajadores, directivos, clientes y proveedores.

3. Elaboración del diagrama causa-efecto. Como parte del trabajo de exploración realizado se llegó a la conclusión que para identificar las posibles causas en las deficiencias observadas era pertinente elaborar un diagrama causa-efecto o diagrama de Ishikawa o espina de pescado.

4. Identificación de los aspectos relevantes en la gestión ambiental: tomando en cuenta los aspectos seleccionados de la revisión documental, los resultados de la observación y del diagrama causa-efecto se identificaron 15 indicadores que resultan importantes para el mejoramiento de la gestión ambiental en entidades de restauración, dígase:

- Estado del edificio. Fachada.

- Entrada del restaurante.

- Iluminación.

- Ventilación y climatización.

- Abastecimiento de agua.

- Consumo de energía.

- Condiciones higiénico-sanitarias.

- Alimentos y bebidas.

- Higiene ambiental.

- Manejo de desechos.

- Control de vectores y animales nocivos.

- Servicios sanitarios públicos.

- Cocina.

- Almacenamiento.

- Requisitos médicos.

En su conjunto, dichos indicadores incluyen un total de 101 aspectos. Díaz (2013) propuso para la evaluación una escala de puntuación valor de -1 (refleja los comportamientos negativos), 0 (comportamientos estancados) y 1 (comportamientos positivos). Para determinar el desempeño se realiza la sumatoria por cada uno de los aspectos, teniendo en cuenta que la escala seleccionada permite con facilidad expresar el estado de los indicadores por cada uno de sus aspectos, pues el aumento de un indicador hacia lo 
positivo refleja lógicamente su comportamiento favorable y por el contrario entre más aspectos tienden hacia lo negativo muestran un desempeño desfavorable y la cantidad de indicadores con aspectos evaluados de 0 determinan el grado de estancamiento o de nula actuación al respecto. Para el análisis integral de la evaluación de la entidad, se considera un desempeño ambiental positivo, si los indicadores evaluados están en el rango entre el 70 y el $84,99 \%$ respecto al total de indicadores (101), y por el contrario cuando los indicadores evaluados reflejan un comportamiento por debajo del $70 \%$, el desempeño de la entidad es negativo.

La aplicación de esta guía permitió la evaluación ambiental sirviendo de diagnóstico en la determinación de aquellos problemas ambientales más críticos para el desarrollo de la misión fundamental de las entidades de ofrecer un servicio de calidad de la actividad gastronómica en la atención y satisfacción a la clientela, y atender y trabajar en aquellos elementos que requieren la adopción inmediata de medidas por parte de la administraciones y los demás implicados. La investigación tomó como muestra tres restaurantes que pertenecen a diferentes grupos empresariales.

\section{ANÁLISIS DE RESULTADOS}

Comportamiento del desempeño ambiental de las entidades objeto de estudio.

Breve caracterización de las entidades:

\section{- Restaurante "El Delfín".}

Pertenece a la Unidad Empresarial de Base (UEB) de Gastronomía del Residencial Tarará SA. de la Corporación Cimex SA. en La Habana. Ubicado en Calle Cobre esq. 4, en el Residencial Tarará, Municipio Habana del Este, Vía Blanca, Km 17, provincia La Habana.

Se inauguró en el año 2002 con el nombre de "Gran Azul" y posteriormente en el año 2006 se reapertura con otras ofertas gastronómicas y se le otorga el nombre de "El Delfín", con la finalidad de prestar servicios de restauración a los pacientes de la Misión Milagro.

Al concluir esta Misión, el restaurante se orienta para fines comerciales, con ofertas gastronómicas que brindan servicios a clientes nacionales e internacionales. En la actualidad se han destinado recursos para mantener la rentabilidad, su correcto funcionamiento y la afluencia de los clientes a la instalación. 
El servicio gastronómico está clasificado como Gastronomía media y se realiza servicio a la Mesa.

Está categorizado operacional y comercialmente como Gastronomía media. Su estado constructivo es bueno. Tiene Licencia Sanitaria.

La fuerza laboral la integran un total de 12 trabajadores, de ellos, administración 1 Servicios generales y de apoyo 1 servicio gastronómico 6 cocina 4 . En el orden profesional se señala que el Administrador es Licenciado en Turismo, y los trabajadores de bar, dependientes gastronómicos, cajeros, cocineros, y luncheros son graduados de Escuelas de Hotelería y Turismo (FORMATUR).

- Restaurante "La Casa Española".

Pertenece al Grupo Empresarial Extrahotelero Palmares S.A. (GEE Palmares) Ubicado en Esquina 7 ma y 26 , Miramar, Playa, La Habana.

Se inauguró en el año 2005, naciendo desde entonces con el nombre de "La Casa Española de la Habana" Hoy es un complejo gastronómico, la fabricación del inmueble donde se encuentra data de la década del cincuenta del pasado siglo. Su estilo arquitectónico, que recuerda una construcción medieval, conserva techos y gran parte del piso originales, en una edificación que posee cuatro estructuras a nivel coronadas por un magnifico mirador. Varios documentos de la época, en especial, la prensa y los libros de la Sociedad Habanera, refieren que el Dr. Gustavo Gutiérrez Sánchez, Ministro que atendía la Economía del país durante el gobierno de Fulgencio Batista, fue quien promovió su construcción como residencia para su familia. Muchos de los materiales para la decoración del lugar y los artistas que trabajaron en la obra fueron traídos desde España. Esto se puede comprobar en la firma que autentifica las losas de la entrada con el nombre del fabricante. Incluye detalles de la decoración que muestran un sentimiento de cubanía como son los vitrales del salón "Mudejar" que representan los escudos de las primeras 6 provincias de la isla.

El servicio gastronómico es de restauración especializada, y donde el visitante podrá degustar platos originales de diversas comunidades españolas con el inigualable toque criollo. Además del servicio a la carta, la casa ofrece diferentes modalidades de contratación como servicio de menú ejecutivo y servicio familiar.

Está categorizado operacional y comercialmente como 3 Tenedores. Su estado constructivo es bueno. Tiene Licencia Sanitaria, aprobado su Sistema de Gestión de la 
Calidad y Aval Medio Ambiental. Cuenta además con múltiples reconocimientos, dentro de ellos, su participación en la Feria Comercial 2015.

La fuerza laboral la integran un total de 29 trabajadores, de ellos, 24 en las áreas de producción, servicios o de actividades fundamentales y 5 como regulación, control y apoyo. En el orden profesional se señala que el Administrador es Licenciado en Turismo, y los trabajadores de bar, dependientes gastronómicos, cajeros, cocineros, y luncheros son graduados de Escuelas de Hotelería y Turismo (FORMATUR).

\section{- El Restaurante "El Cochinito".}

Pertenece a la Empresa Restaurantes Habana del Poder Popular .Ubicado en Calle 23 entre H e I Vedado, Municipio Plaza de la Revolución.

Fue inaugurado por Celia Sánchez Manduley el 25 de Diciembre de 1965 quien, por iniciativa propia, y con la intención de recrear en el centro del Vedado un ambiente típicamente campestre y contribuir con el propósito del Gobierno Revolucionario desde ese entonces de lograr la fusión entre ambas culturas, el campo y la ciudad, y para el disfrute de nuestro pueblo trabajador y el turismo en general, fundó este emblemático sitio. Con una arquitectura de casa colonial y ambientado con jardines y plantas propias de la flora nacional que combinan con un mobiliario rústico conformado por taburetes de cuero y mesas de madera unido a otros elementos decorativos que imitan un escenario campestre, se brinda una imagen que inspira al cliente a disfrutar de una oferta gastronómica especializada, amenizada con música tradicional cubana.

El Servicio Gastronómico utilizado es uno de los más sencillos y se conoce como servicio "A la Española" donde los alimentos se sirven en fuentes y soperas para que el propio cliente se sirva a gusto los deliciosos platos que se elaboran a partir de la carne de cerdo y otros platos de la cocina cubana siendo la misma un vivo reflejo de la identidad cultural nacional.

Está categorizado operacional y comercialmente como Gastronomía media. Su estado constructivo es bueno. Tiene Licencia Sanitaria.

La fuerza laboral la integran un total de 56 trabajadores, de ellos, Administración 5 Servicios generales y de apoyo 11 servicio gastronómico 25 cocina 15. En el orden profesional se señala que el Administrador es Licenciado en Turismo, y los trabajadores de bar, dependientes gastronómicos, cajeros, cocineros, y luncheros son graduados de Escuelas de Hotelería y Turismo (FORMATUR). 
Resultados comparados del Desempeño ambiental:

Análisis derivados de la aplicación de la GOA según entidades seleccionadas

Restaurante "La Casa Española de la Habana": A la entidad se le otorga la "Evaluación Integral de Bien", para su desempeño ambiental, porque cumple las condiciones siguientes: Tiene 76 indicadores evaluados de positivo, para el $74,50 \%$ ( 70 a $84,99 \%$, requisito para Bien) respecto al total de indicadores (101). Además cumple los requisitos de poseer Licencia sanitaria, Aval Ambiental y estar Categorizado. Ello es evidencia de que la política ambiental del Grupo Empresarial Extrahotelero Palmares S.A. (GEE Palmares) está orientada hacia el perfeccionamiento de la gestión ambiental de las entidades bajo su gerencia. El Objetivo general de esta Política se basa en garantizar la calidad y el respeto al medio ambiente de sus productos y servicios, asegurando la conformidad de los mismos con las exigencias, especificaciones y requisitos establecidos en las normas que regulan la protección del medio ambiente, de modo tal que armonice el empleo eficaz de las potencialidades de los recursos naturales, con la garantía que puedan proporcionar iguales ó superiores beneficios a las generaciones futuras, respetando la cultura Nacional y sus expresiones territoriales, contribuyendo así a la elevación de la calidad de vida.

Restaurante "El Delfín ": La entidad en estos momentos tiene un desempeño ambiental negativo teniendo en cuenta que de los 101 aspectos evaluados, 60 manifestaron desempeño negativos en contraste con solo 41 con desempeños positivos. Las situaciones más críticas están relacionadas con los indicadores vinculados a: el estado del inmueble, abasto de agua, condiciones higiénico - sanitarias, alimentos y bebidas, higiene ambiental, manejo de desechos, cocina y almacenamiento.

Restaurante "El Cochinito": Al igual que en el caso anterior, la entidad en estos momentos tiene un desempeño ambiental negativo. Teniendo en cuenta que de los 101 aspectos evaluados, 55 manifestaron comportamientos negativos en contraste con solo 46 con resultados positivos.

Entre los problemas ambientales está principalmente la contaminación ambiental asociada a las dificultades higiénicos sanitarios, al no contar con estabilidad permanentemente en los aseguramientos de productos para desinfección e higiene, no existe una política consecuente encaminada al tratamiento y reducción de residuales con alternativas biodegradables y en consecuencia debe elaborarse un programa de ahorro de agua, electricidad y clasificación de desechos. 
Valoración integral de los resultados

En las entidades analizadas las áreas con desempeños negativos, se asocian a los indicadores vinculados a: el estado del inmueble, abasto de agua, condiciones higiénico sanitarias, alimentos y bebidas, higiene ambiental, manejo de desechos, cocina y almacenamiento. Este desempeño está determinado por los problemas higiénicos sanitarios, al no contar con estabilidad permanentemente en los aseguramientos de productos para desinfección e higiene, no existe una política consecuente encaminada al tratamiento y reducción de residuales con alternativas biodegradables y en consecuencia debe elaborarse un programa de ahorro de agua, electricidad y clasificación de desechos. El desempeño positivo estuvo asociado a los aspectos relacionados con la entrada del restaurante, la iluminación, el control de vectores, los servicios sanitarios públicos y los requisitos médicos.

Resultando estancados los indicadores de ventilación y consumo de energía.

\section{Propuesta para el mejoramiento del desempeño ambiental de entidades de restauración del destino La Habana.}

Los Restaurantes "El Delfín" y "El Cochinito" deben trabajar para lograr significativos resultados en su desempeño ambiental ya que se requiere de una adecuada comercialización del producto turístico con altos niveles de competitividad y sostenibilidad en la gestión organizacional, todo lo cual hace necesario la aplicación integradora de las Normas Cubanas ISO 14000 y con ellas un adecuado cumplimiento de la Legislación Ambiental.

Teniendo en cuenta estas premisas, se propone que las acciones de dichas entidades deben estar dirigidas a definir los Objetivos de trabajo para la Gestión Ambiental, acompañadas de un grupo de tareas principales para llevar a vías de cumplimiento los mismos. Sobre la base de ambas acciones se sustentará el resto de las actividades que deben desarrollarse pues son dependientes de las que se listan a continuación.

Objetivos

1. Comenzar la aplicación de un Sistema de Gestión Ambiental a partir de la Norma Cubana ISO 14001

2. Establecer un sistema de capacitación, divulgación e Información en materia de Gestión Ambiental. 
Tareas principales

1. Constituir la Comisión de base de Medio Ambiente.

2. Realizar un Diagnóstico Ambiental.

3. Definir la Política Ambiental.

4. Elaborar un Programa de Gestión Ambiental para dar solución a las no conformidades definidas en el Diagnóstico.

5. Elaborar un Programa de actividades para dar cumplimiento a los Objetivos y Tareas definidas.

6. Definir en el presupuesto todas las partidas que llevan implícito acciones para la Gestión Ambiental.

7. Gestionar los cursos que se encuentran disponibles sobre Medio Ambiente y que deben recibir los especialistas y directivos para su formación.

8. Organizar con los especialistas y directivos capacitados un sistema de divulgación, información y capacitación interno para elevar la cultura ambiental de todos los trabajadores y clientes.

9. Vincular estrechamente todas las actividades de la Gestión Ambiental con el Fórum de Ciencia y Técnica, así como con los Sistemas de Calidad y Seguridad.

10 Constitución de la comisión de medio ambiente.

Será responsabilidad de esta comisión establecer y controlar el cumplimiento de la Política, Objetivos y Tareas para la Gestión Ambiental de los restaurantes en cuestión. Se adiciona la responsabilidad de establecer y controlar el cumplimiento de la legislación ambiental nacional, así como trabajar para el logro de los reconocimientos asociados a la gestión ambiental nacional e internacional.

Debido a que ni la Corporación Cimex, ni la Empresa Restaurantes Habana del Poder Popular de La Habana tienen definida una política ambiental esto trae como consecuencia que sus entidades no sean capaces de cumplir correctamente con las regulaciones establecidas. Por esta razón se deben tomar en cuenta los siguientes aspectos para trazar una política que esté encaminada a:

- Tener como prioridad principal el incremento de la educación y la cultura medioambiental de los trabajadores y clientes de la instalación

- Comenzar la formación y aplicación de un Sistema de Gestión Ambiental a partir de las Normas ISO 14000, que debe ser orientado a la mejora continua e integrar con las actividades de Calidad y Seguridad para obtener resultados eficientes sin producir afectaciones significativas al medio ambiente 
- Procurar una gestión de compras para la adquisición de productos, insumos y tecnologías amigables con el medio ambiente

- Tener en cuenta el estricto control sobre el uso eficiente de los recursos disponibles, las materias primas, el agua y los portadores energéticos, así como de todos los desechos.

- Incorporar la actuación en procesos que sean cada vez más limpios en aras de poder brindar productos y servicios a los clientes que permitan incrementar su nivel de satisfacción.

\section{CONCLUSIONES}

La implantación de sistemas de gestión ambiental en las empresas turísticas es una necesidad en tanto se traduce como valor agregado para las organizaciones y tributa no solo al perfeccionamiento de su gestión sino que les permite disponer de ventajas competitivas en un entorno que tanto para clientes como para prestatarios precisa de ofertas eco responsables en ambientes seguros.

El diagnóstico conllevó a evaluar como deficiente en su desempeño ambiental a los restaurantes El Delfín y El Cochinito mientras que La Casa Española fue catalogada como bien al sobresalir en las actuaciones positivas en torno a su gestión ambiental.

Los restaurantes evaluados mantienen actuaciones ambientales positivas similares en indicadores relacionados con la entrada al inmueble, la iluminación, control de vectores y animales nocivos y requisitos médicos, mientras que hay incidencias negativas en el manejo de desechos sólidos y la cocina.

Las acciones propuestas, en consonancia con los aspectos evaluados y las deficiencias identificadas están encaminadas a la definición de objetivos y tareas, la constitución de la comisión de medio ambiente en las entidades y los aspectos para trazar una política ambiental así como al perfeccionamiento de la definición, enfoques, objetivos y estrategias ambientales desarrolladas.

\section{REFERENCIAS BIBLIOGRÁFICAS}

Águila, María E. Evaluación del desempeño ambiental del restaurante El Delfín. Trabajo de diploma en opción al título de licenciado en turismo. Facultad de Turismo, Universidad de La Habana. 2015. 
Almeida, A., Sistema de gestión ambiental bajo el modelo ISO 1400. Facultad de Ciencias Administrativas y Recursos Humanos. Universidad de San Martín de Porras. Lima. Perú. 15-16. 2004.

Arce, M., Diagnóstico de los Sistemas de Gestión Medio Ambiental y su impacto en los hoteles del Polo turístico de Santa Lucía. Tesis en opción del grado de Máster en Gestión Turística. Universidad de Camagüey. Cuba. 18. 2007.

Ayala, M. y Gárciga, J., Diagnóstico ambiental, punto de partida hacia el Sistema de Gestión Ambiental. Centro de Gestión y Desarrollo de la Calidad (CGDP). La Habana. Cuba. (1) 37- 40. 2006.

Ayes, G., Medioambiente, impacto y desarrollo. Editorial Científico Técnica, La Habana. Cuba. 179. 2003.

Bacallao, A. La educación ambiental: retos y desafíos en la formación académica de los futuros policías, Trabajo final en opción al título de especialista en trabajo social comunitario, Universidad de La Habana. 2010.

Cabanes, C. Evaluación del desempeño ambiental del restaurante El Cochinito. Trabajo de diploma en opción al título de licenciado en turismo. Facultad de Turismo, Universidad de La Habana. 2014.

Centro de Información, Gestión y Educación Ambiental (CIGEA). Estrategia de introducción del concepto de la Producción más limpia en la gestión ambiental empresarial Ministerio de Ciencia Tecnología y Medio Ambiente (CITMA). La Habana. Cuba. 27-29. 2002.

CITMA. Estrategia Ambiental Nacional (EAN) 2011-2015. La Habana.

CITMA-MINTUR. Convenio de cooperación CITMA-MINTUR. Documento de trabajo. 2003. 
Díaz, L. Evaluación del desempeño ambiental del restaurante "La Vicaria”, Trabajo de diploma en opción al título de licenciado en turismo, Facultad de Turismo, Universidad de La Habana.2013.

González, M.L., Gestión Ambiental de los Impactos del Turismo en Espacios Geográficos Sensibles. Ediciones Abya-Yala. Quito. Ecuador. 56-58, 2006.

Gutiérrez, O., Cuba, turismo y desarrollo económico. Centro de Estudios de la Economía Cubana. Universidad de La Habana. Cuba. (2000). Disponible en: Página web: Cuba siglo XXI, Economía (13 de octubre del 2012).

Hernández, M., Gestión empresarial. Desafíos y oportunidades desde la perspectiva de la gestión ambiental. Revista trimestral Latinoamericana y Caribeña de Desarrollo Sustentable (revista electrónica). Ediciones Revista Futuros. Canadá. (17). 2007.

Ley No. 81 del Medio Ambiente. Asamblea Nacional Del Poder Popular. Ministerio de Ciencia, Tecnología y Medio Ambiente (CITMA)]. Capítulo II. Aspectos básicos. Gaceta Oficial de la República de Cuba, edición extraordinaria. La Habana. Cuba. 1997.

Mateo, J., Referenciado en Turismo y Medio Ambiente: Apuntes. Facultad de Geografía. Universidad de La Habana. Cuba. 31-32. 2002.

Marín, K. Evaluación del desempeño medio ambiental en el restaurante "La Casa Española" perteneciente a Sucursal Extrahotelera Palmares Centro S.A. Trabajo de diploma en opción al título de licenciado en turismo. Facultad de Turismo, Universidad de La Habana, 2015.

Marx, Carlos: El capital, tomo I. Editorial de Ciencias Sociales. La Habana, 1973.

Maza, C., Manejo y conservación de recursos naturales. Editorial Universitaria. Barcelona. España. 508, 2009.

MINTUR. Estrategia Ambiental Nacional del MINTUR 2012-2015. 
NC ISO 14000: 1997. Sistema de Gestión Ambiental. Oficina Nacional de Normalización. La Habana. Cuba, 1997.

NC ISO 14001:2004. Sistemas de Gestión Ambiental. Requisitos con orientación para su uso. Oficina Nacional de Normalización. La Habana. Cuba, 2004.

NC ISO 14015: 2005. Gestión Ambiental. Evaluación ambiental de sitios y organizaciones (EASO). Directrices. Oficina Nacional de Normalización. La Habana. Cuba, 2005.

NC ISO 14031: 2005. Gestión Ambiental. Evaluación del Desempeño Ambiental. Directrices. Oficina Nacional de Normalización. La Habana. Cuba, 2005.

NC ISO 14050:2005. Gestión ambiental - Vocabulario. Oficina Nacional de Normalización. La Habana. Cuba, 2005.

Perspectivas del Medio Ambiente Mundial, Sistema de Gestión Ambiental. Producciones limpias. Publicado por el Programa de las Naciones Unidas para el Medio Ambiente, PNUMA. 36, 2000.

Perelló, J.L., Introducción a la Investigación de Mercados Turísticos. Centro de Estudios Turísticos. Universidad de La Habana. Cuba. 36, 2005.

Tang, C.F. y Abosedra, S., (2014). The impacts of tourism, energy consumption and political instability on economic growth in the MENA countries. Energy Policy, 68, pp.458-464.

Tejeda, M.; Rodríguez, A. y Otros., Concepción teórico metodológica para la aplicación práctica de los Sistemas de Gestión Ambiental en Organizaciones Turísticas. EHTH. La Habana. Cuba. 47-48, 2010.

Yasarata, M., et al. (2009): "Politics and sustainable tourism development - Can they coexist? Voices from North Cyprus". Tourism Management xxx, pág. 1-12. 
Licencia Creative Commons

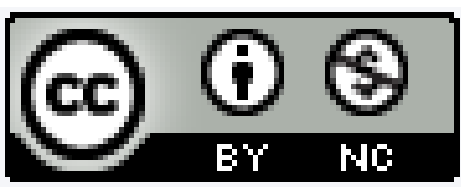

Revista Científica ECOCIENCIA está bajo una Licencia Creative Commons Atribución-NoComercial 4.0 Internacional. 